\title{
UNA MIRADA A LA EVAPORACIÓN COMO OPERACIÓN EN LA INDUSTRIA QUÍMICA
}

\author{
A LOOK AT EVAPORATION AS AN OPERATION IN THE CHEMICAL INDUSTRY
}

Luis Eduardo Ayala Padilla*

https://orcid.org/0000-0001-9038-317X

John Antonny Pabón Beltrán*

https://orcid.org/0000-0002-8534-7497

Felipe Correa-Mahecha*

https://orcid.org/0000-0003-1050-8222

FUNDACIÓN UNIVERSIDAD DE AMÉRICA

Recibido: 2 de septiembre de 2018

Aceptado: 24 de junio de 2019

DOI: https://doi.org/10.29097/23461098.304

\section{Resumen}

La evaporación como operación unitaria ha cobrado gran importancia en la industria debido a que puede disminuir los costos del transporte de fluidos sin alterar las propiedades fisicoquímicas de la sustancia. Este artículo hace una revisión bibliográfica acerca de los diferentes tipos de evaporadores de uso común en la industria, así como de los principales factores que han sido estudiados para mejorar su eficiencia. Igualmente, aquí se trata sobre el modelamiento y simulación de estos equipos para realizar procesos de optimización y sobre el uso de nuevas tecnologías para ampliar el campo de aplicación de dicho procedimiento.

Palabras clave: evaporación, tren de evaporación, eficiencia, modelamiento y simulación, nuevas tecnologías para la evaporación.

\section{Abstract}

Evaporation as a unit operation has gained great importance in the industry due to the need to reduce transport costs without altering the physicochemical properties of the fluid, which is why this work focuses on making a bibliographic sweep of the different types of Many evaporators used in the industry, as well as the main factors that have been studied to improve their efficiency. On the other hand, different authors who carry

\footnotetext{
* Ingeniero químico. Investigador, grupo de investigación Procesos de separación no convencionales, línea de investigación Procesos de separación sin reacción.

** Ingeniero químico. Coinvestigador, grupo de investigación Procesos de separación no convencionales, línea de investigación Procesos de separación sin reacción.

*** Ingeniero químico. Docente investigador, grupo de investigación Procesos de separación no convencionales. $\boldsymbol{\text { felipe.correa@profesores.uamerica.edu.co }}$
} 
out modeling and simulation of these teams are enunciated in order to carry out optimization processes and, finally, the scientific advances that have reached this unitary operation in the industry framed in the new technologies applied in said operation.

Keywords: evaporation, evaporation train, efficiency, modeling and simulation, new technologies for evaporation.

\section{INTRODUCCIÓN}

La evaporación es una de las operaciones unitarias más importantes de la ingeniería química. Esta técnica se emplea en diferentes industrias, especialmente para la concentración de sustancias (soluciones acuosas de azúcar, cloruro de sodio, hidróxido de sodio, glicerina o jugos de frutas) (Rojero, García, Serrano, Félix y Ríos, 2008). El proceso consiste en la adición de calor a una solución para evaporar el disolvente, que generalmente es agua o un compuesto orgánico. En la mayoría de los casos la solución concentrada es el producto deseado y el solvente (agua) evaporado suele desecharse (Geankoplis, 1998). La mayoría de los evaporadores se calientan con vapor de agua, el cual se condensa sobre tubos metálicos.

Durante la evaporación del solvente la concentración de la solución crece y propiedades como la densidad y la viscosidad aumentan con el contenido de sólidos hasta que la solución se transforma en saturada o resulta inadecuada para una correcta transferencia de calor (García, Peña, López, Duran y Olvera, 2009). Con frecuencia el líquido que hierve se encuentra a un vacío moderado para reducir su temperatura de ebullición, lo que aumenta la diferencia de temperatura entre el vapor condensante y el líquido de ebullición y, por tanto, aumenta la velocidad de transmisión de calor en el evaporador (Seader, Henley y Roper, 2011). Existen diferentes tipos de evaporadores, cada uno con sus respectivas aplicaciones y especificaciones de uso. A continuación, se realiza una revisión bibliográfica sobre los tipos de evaporadores, con el fin de dar al lector una herramienta que permita conocer de primera mano estos equipos. El propósito de este artículo es realizar una compilación bibliográfica que sirva de guía para todos aquellos interesados en conocer cómo va la industria de la evaporación, ya que, según el análisis de Scopus, solo el 3.4 \% de los artículos publicados son de revisión o resúmenes de conferencias, lo cual dificulta la selección de un tema de investigación al respecto.

\section{METODOLOGÍA}

Este documento parte de la construcción de un contexto acerca de la evaporación, entendiendo que esta es una operación unitaria de relevancia en la industria. El artículo trata de los diferentes tipos de evaporadores, realiza una descripción y enumera las diferentes aplicaciones. Para esto se recurrió a la recolección, procesamiento y análisis de información de fuentes secundarias provenientes de bases de datos científicas (ScienceDirect, Virtual Pro, SpringerLink, ResearchGate) y repositorios universitarios (Universidad Nacional de Colombia, Universidad de Córdoba y Fundación Universidad de América). Al respecto ver Figura 1. 


\section{Figura 1}

Fuentes bibliográficas consultadas.

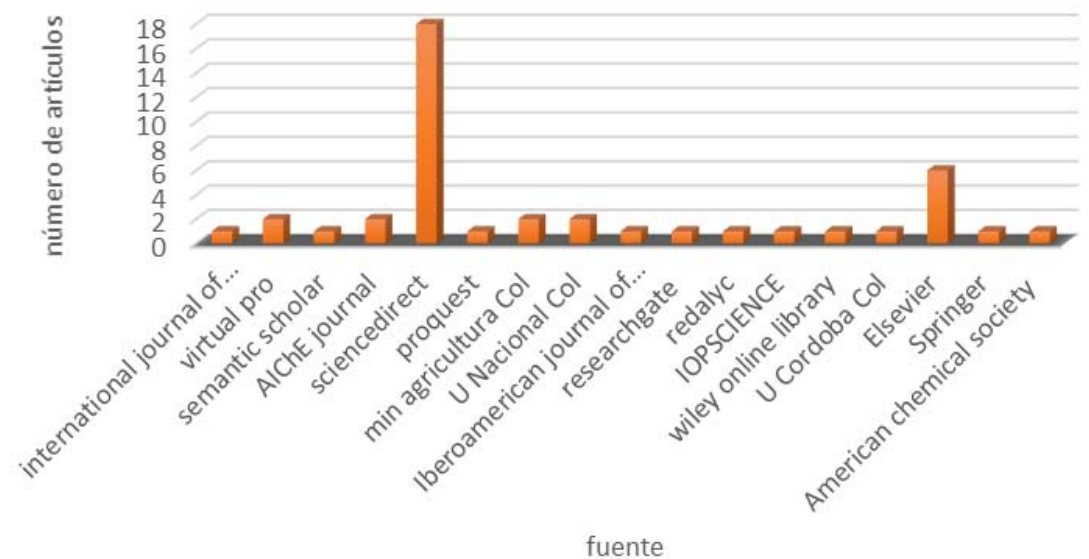

Nota. De acuerdo con la gráfica, queda en evidencia que ScienceDirect es el repositorio que más aportó al presente artículo, con 18 publicaciones de un total de 43.

De acuerdo con las herramientas bibliométricas de Scopus, existen alrededor de 500 artículos relacionados con la operación de evaporación en trenes multiefecto, de los cuales el $10 \%$ se encuentran publicados en la revista Desalination. Las demás fuentes se muestran en la Figura 2.

\section{Figura 2}

Revistas de investigación que publican artículos de evaporación.

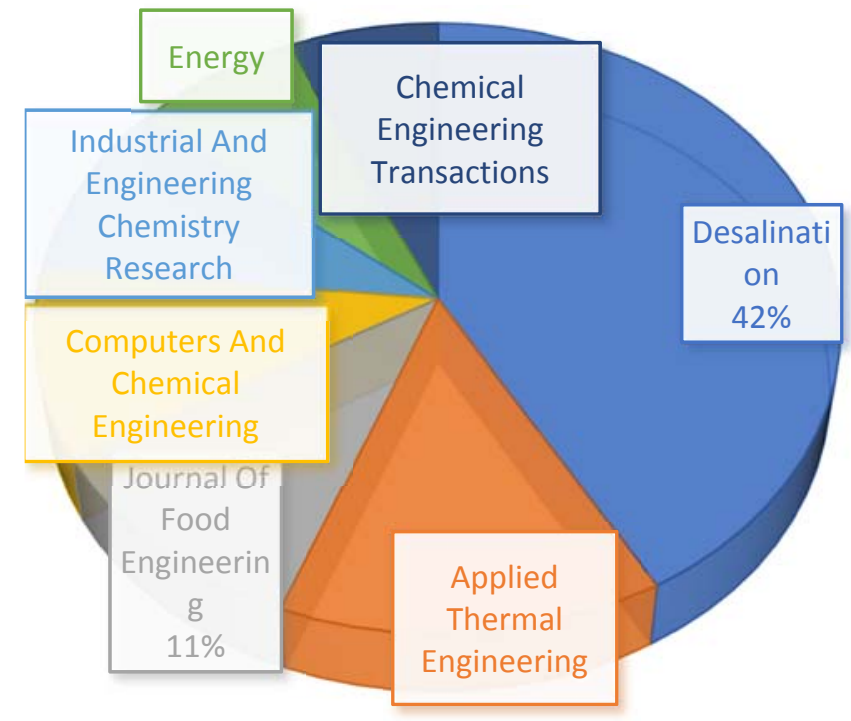

Nota. Datos extraídos de Scopus (2018). 
Los países que más han aportado en el tema de la industria de la evaporación son Estados Unidos, con 81 artículos, y la India, con 59 publicaciones. El aporte de los demás países se representa en la Figura 3. Se evidencia que la India es actualmente el abanderado en el tema, en particular, las investigaciones del Indian Institute of Technology Roorkee, con 18 publicaciones y énfasis en la industria azucarera.

\section{Figura 3}

Producción por países de artículos referentes a evaporación.

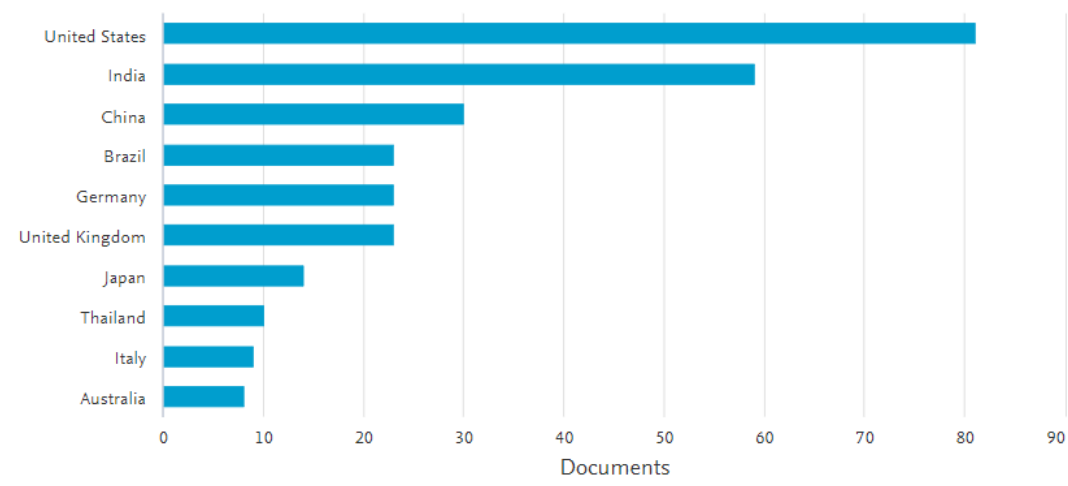

Nota. Datos extraídos de Scopus (2018).

Observamos en la Figura 4 los principales autores que han tratado el tema de la evaporación. Un tailandés y dos hindús son los autores con más publicaciones (8 cada uno).

Figura 4

Principales autores que tratan el tema de evaporación.

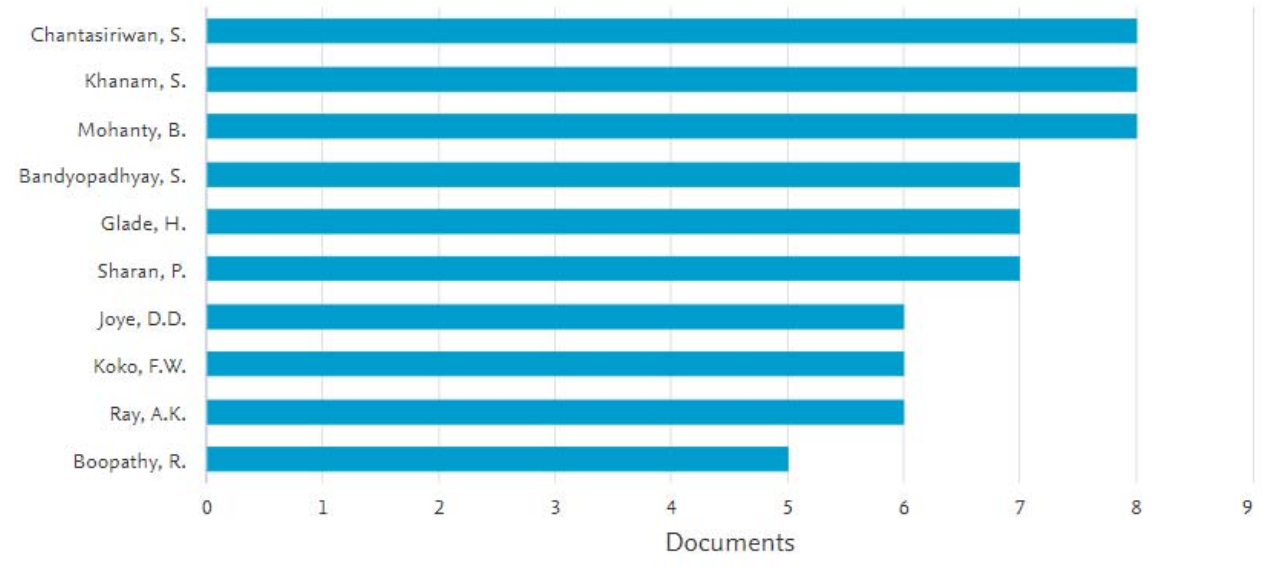

Fuente: Datos extraídos de Scopus (2018). 


\section{RESULTADOS}

\section{Los evaporado res en la industria química}

De acuerdo con la revisión bibliográfica, el evaporador de marmita abierta es uno de los primeros dispositivos creados para eliminar el contenido de agua de la solución a concentrar. Consiste en un recipiente cilíndrico, el cual se pone en contacto directo con una fuente de energía para que realice la transferencia de calor y se evapore el solvente volátil.

Actualmente, dicho evaporador ha tenido mejoras circunstanciales en cuanto al método de calentamiento, como el uso de una resistencia eléctrica o una chaqueta de calentamiento, las que calientan el sistema y permiten la evaporación del agua presente en la mezcla. Padilla (2013) realizó el diseño de un equipo con el fin de satisfacer las necesidades que la Universidad de San Carlos de Guatemala, en cuanto a "la elaboración de diferentes productos alimenticios como manjares, jaleas, mermeladas, salsas, jugos y pasteurizaciones en general” (p. XVI).

\section{Figura 5}

Evaporador de película descendente.

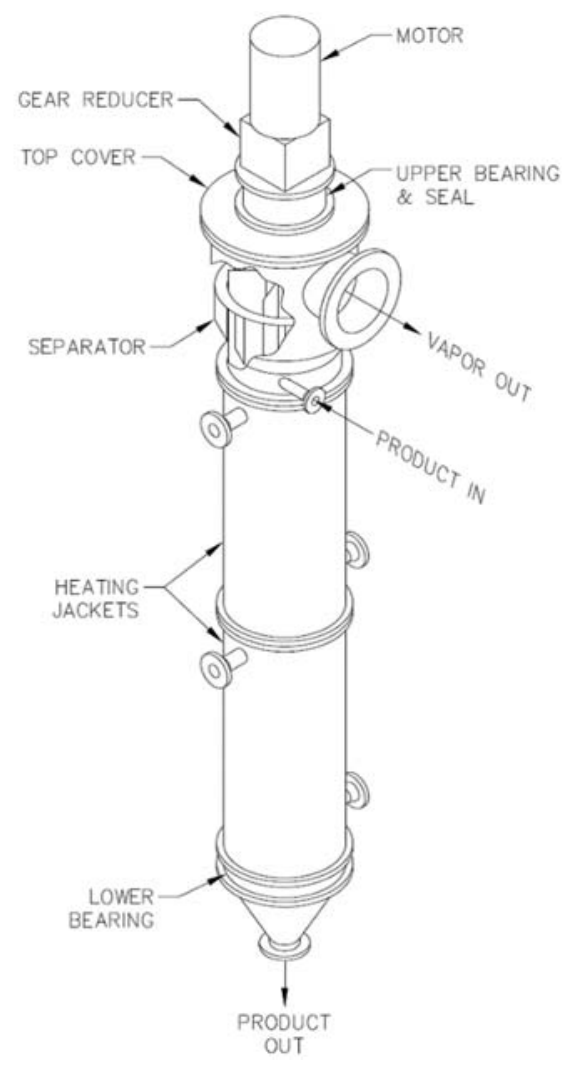

Fuente: Jastoria (2015). 
La principal falencia que tiene este equipo es el no aprovechamiento del vapor generado en la evaporación del solvente, lo que implica que la economía del proceso sea pobre. Por otra parte, la velocidad de transferencia de calor es baja, ya que el área de transferencia de calor está limitada a las paredes del evaporador. Considerando estas desventajas, Padilla (2013) realizó la instalación de agitadores, no solo para mejorar la velocidad de transferencia de calor, sino también para evitar que el fluido se queme.

Comúnmente se instalan serpentines para aumentar el área de transferencia de calor entre las corrientes; pero, según Brennan, Butters y Lilly (1998), "los serpentines de calentamiento internos que se instalan en las unidades mayores pueden dificultar la circulación del líquido y, por tanto, reducir la velocidad de transferencia de calor” (p.354), lo que indica que el uso de estos evaporadores está condicionado al tamaño del equipo.

El evaporador de película descendente (véase Figura 5) es usado para realizar la concentración de sustancias en las que las propiedades se ven afectadas por el tiempo de transferencia de calor, como es el caso de la industria láctea, ya que al mantenerse por periodos prolongados se crea una capa que se adhiere a las paredes del evaporador, no solo ocasionando la formación de incrustaciones, sino perdiendo además gran cantidad de solidos disueltos en la leche (Gourdon y Mura, 2017).

La industria azucarera está tecnificando los procesos de obtención de panela. Se han dejado de lado los sistemas tradicionales, en los que se hace uso de "pailas” y no se aprovecha el agua que es evaporada en el sistema. La India abandera la tecnificación de dicho proceso, país en el que se tratan temas relacionados con: determinación de coeficientes de transferencia de calor; área de transferencia de calor (Chantasiriwan, 2018); simulaciones robustas del proceso de producción de la panela, aplicando conceptos de integración energética y haciendo uso del análisis pinch para su ejecución (Jyoti y Khanam, 2014); caracterización de un evaporador (Srivastava, Mohanty y Bhargava, 2016); configuración más adecuada para el proceso de concentración del jugo de caña (Gautami y Khanam, 2012), entre otros aspectos. Colombia está adelantando estudios en cuanto al uso de esta tecnología para hacer más competitivo y rentable este proceso de producción. Al respecto, Mendieta, Rodríguez y García (2008), en un estudio desarrollado por Corpoica, utilizan el concepto de trenes de evaporación (ver Figura 6) con el fin de realizar el aprovechamiento del vapor generado en cada etapa del proceso de evaporación y así disminuir los costos de operación.

Los autores diseñaron un sistema de múltiple efecto, el cual es provisto de evaporadores verticales de canasta con circulación natural o forzada, ya que la viscosidad del fluido aumenta en función de la cantidad de agua removida, por esto el fluido a concentrar viaja por la coraza, mientras que el vapor circula por los tubos (Seader et ál., 2011). 
Figura 6

Tren de evaporación de triple efecto.

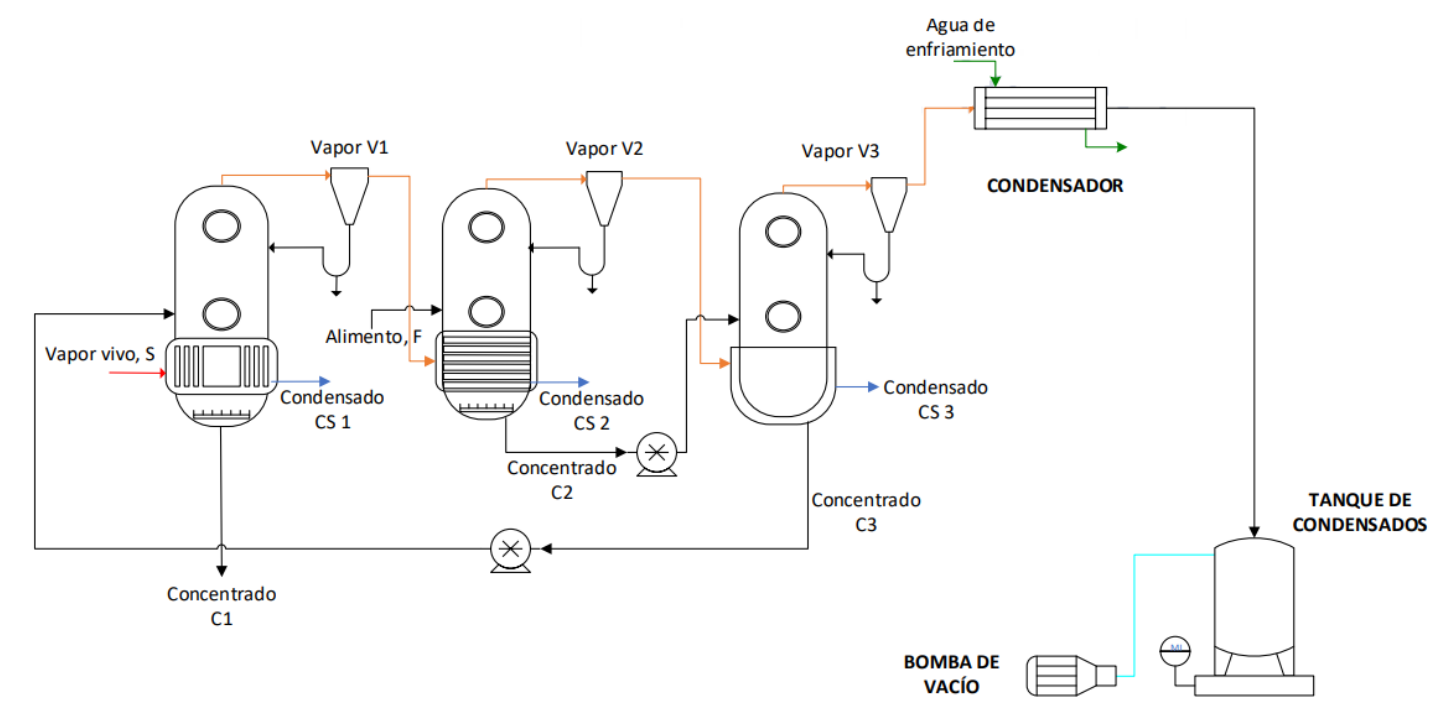

Fuente: Process Solutions and Equipment (2018).

Los evaporadores son ampliamente utilizados en la industria alimenticia. Al respecto, es necesario hacer una precisa caracterización al fluido que ingresará al evaporador, con el fin de seleccionar el evaporador pertinente para realizar la operación. El estudio de Avalo, Pérez y Tovar (2009) presenta la caracterización del jugo de naranja, en la que se midieron parámetros iniciales, como cantidad de sólidos solubles totales presentes en la solución, cantidad de azúcares reductores (y no reductores) y pH.

Gracias a la caracterización realizada se seleccionó un tren de evaporación de triple efecto, conformado por evaporadores de calandria vertical con tubos cortos, en los que se desarrolló la operación con el fin de obtener la mayor cantidad de vitamina C presente en la solución concentrada, lo que aumentó la viscosidad de $12{ }^{\circ} \mathrm{Bx}$ a $25.5^{\circ} \mathrm{Bx}$ en 164 $\min$.

En el proceso de concentración de jugo de tomate en un evaporador de doble efecto se evidencia cómo se selecciona el tipo de evaporador según las necesidades de preservación de las propiedades del fluido a concentrar, en este caso, el contenido de licopeno que proporciona el color rojo. En los análisis de sensibilidad se muestra que se pueden hacer procesos de mejora a la operación modificando las siguientes variables: presiones en cada uno de los evaporadores, capacidad de cada evaporador, cantidad de vapor vivo que entra al sistema y temperatura del sistema (Núñez, Tarifa y Farfán, 2012).

La evaporación como operación unitaria fue utilizada alrededor de 10 años por las industrias israelís (Semiat, 2001). Ante la escasez de agua potable en la región, se han realizado investigaciones relacionadas con la desalinización. Este es un proceso en el 
que se usa la evaporación del agua para separar la sal presente en el agua de mar, adaptándola para el consumo humano. Con el paso del tiempo la desalinización por el método de evaporación ha venido disminuyendo hasta llegar a tan solo ocupar el $2 \%$ de la industria del país. El principal motivo de esta reducción radica en el costo excesivo de energía que se debe suministrar a la solución para obtener un agua potable. Además, la ósmosis inversa desplazó este proceso. Otro factor es el costo de instalación de los equipos en lugares tan distantes, ya que se debe contratar personal especializado para realizar el montaje en dichos lugares y el costo del transporte aumenta (Sánchez y Pérez, 1976). Es por esto que se diseñó un evaporador compacto e independiente en su construcción, con el fin de facilitar el montaje en sitios inhóspitos sin que se requiera personal con alta capacidad técnica para su ensamble.

En la industria petrolera se realiza un tratamiento de evaporación a las aguas de producción, las cuales tienen un alto contenido de $\mathrm{NaCl}$. Por medio de la evaporación se logra grandes resultados. Así, gracias al aprovechamiento de los gases de combustión que se encuentran en el yacimiento, se logra producir el vapor vivo necesario para evaporar el agua presente en la solución a concentrar, lo cual hace que el costo del proceso de evaporación disminuya. Producto de esta operación se han logrado porcentajes de remoción de agua superior a 80 \% e inferior al 92 \%. La concentración de sales alcanza su mayor punto con una pureza cercana al 97 \% (Guerrero, Escobar y Ramírez, 2005).

Igualmente, en la industria del ácido fosfórico se hace uso de la evaporación para la obtención de agua de servicio y enfriamiento en dicho proceso (Harrison, 2014).

\section{Factores por tener en cuenta al mejorar la eficiencia de la evaporación}

Las incrustaciones causadas por la formación de una película de material adherido a las paredes del evaporador es un problema que debe ser atendido de manera oportuna, ya que actúa como una resistencia a la transferencia de calor, lo que ocasiona un aumento en los requerimientos energéticos para evaporar la misma cantidad de solvente. El factor de humectación, entendido como el flujo másico que baña y pasa por los tubos, ayuda a contrarrestar las incrustaciones; para esto se añade un plato de distribución en la alimentación, cuya función es la distribución homogénea (ayudada por la fuerza gravitatoria) del alimento por cada tubo. Morison (2015) diseñó un simulador que puede servir como herramienta de predicción de condiciones óptimas de operación, además de que da al ingeniero los parámetros para realizar el diseño de equipo.

Una vez las incrustaciones son producidas en el evaporador, estas se deben eliminar por medio de paradas técnicas en las que se limpia el equipo a profundidad. Gracias al uso de un planteamiento operativo de redes de operación se logra determinar (mediante un modelo de optimización multiperiodo) la secuencia de paradas de un tren de evaporación usado en la industria azucarera, de acuerdo a los requerimientos que el equipo solicite y manteniendo una alta eficiencia (Heluane, Blanco, Hernandez y Bandoni, 2013). En esta función se tiene en cuenta el costo operativo del sistema que se ve afectado por la disminución de la eficiencia, que es causada por un aumento en la 
resistencia global de transferencia de calor. Al desarrollar el modelo se encontró que se deben realizar dos paradas semanales (cada 5717 ton/h) para limpieza profunda y paradas con un menor grado de limpieza cada 13 horas.

Otro de los parámetros a tener en cuenta es la separación que existe entre el haz de tubos, así como los tipos de tubos (lisos, aletas dimensionales mejoradas 2D y 3D) presentes en el evaporador, los que inciden en el modo de flujo intertubular. Hay tres patrones principales de flujo intertubular en el proceso de caída de película: modos de gota, columna y hoja; con estos patrones y los diferentes tipos de tubos y espaciados internos se determinan los tipos de flujo. Posteriormente, cambiando la velocidad de flujo, se mostró el efecto del espaciado en el número de Re.

El resultado obtenido muestra las diferentes combinaciones entre espaciado y el tipo de tubo, con el fin de lograr el aumento del número de Re, el cual caracteriza el movimiento del fluido. En este caso conviene que el movimiento sea desordenado, ya que el choque de las moléculas presentes en el fluido favorece la transferencia de calor cuando se hace una convección forzada, lo que muestra que el espaciamiento únicamente afecta a los tubos mejorados 3D, ya que el número de Re aumenta. En los demás tubos se evidencia una disminución de Re, lo cual no es favorable (Chen, Zhang, Hu y Мa, 2017).

La caída de presión se debe tener en cuenta a la hora de mejorar la eficiencia. Esta es causada por la fricción entre las partículas presentes en el fluido y las paredes de los tubos del evaporador. Mura y Gourdon (2017) determinaron que el factor de humectación, la velocidad de alimentación y el contenido de solidos secos en el alimento afectan la caída de presión; asimismo, lograron disminuir esta caída con la humectación adecuada a los tubos.

El coeficiente de transferencia de calor en el proceso de evaporación cobra gran relevancia en la operación unitaria, ya que se traduce, en palabras puntuales, como la facilidad con la que se da la transferencia de calor, lo que genera una disminución en la cantidad de energía que se suministra al sistema.

Demesa, Huicochea, Hernandez y Siqueiros (2017) presentan una serie de correlaciones reunidas de manera estratégica para realizar el cálculo del coeficiente global de transferencia de calor, entre las que se destacan el factor de ebullición sobre el cilindro, el calor latente y, por consiguiente, el coeficiente local para la piscina de ebullición. De igual manera, los autores realizaron la misma compilación para la sección interna de los tubos y propusieron un método para calcular la media logarítmica de las temperaturas en la evaporación. Se realizaron diferentes pruebas en las que se varió la temperatura de entrada al evaporador de la fuente de calor, así como el flujo másico del mismo, con el fin de obtener la mayor cantidad de calor transferida en el proceso. Como resultado se obtuvo que la mejor combinación de estos dos factores (temperatura y flujo másico) es $334.35 \mathrm{~K}$ y $0.11 \mathrm{~kg} / \mathrm{s}$, que implica un Q de $2 \mathrm{~kW}$, lo cual impacta directamente en la economía del proceso, es decir, produce más energía con menos cantidad de alimento. 
Los factores anteriores deben ser tenidos en cuenta para mejorar la eficiencia del proceso y estar ligados a la preservación de las propiedades del fluido a evaporar; por ejemplo, el sabor en la leche en polvo que se ve afectado por la disminución de radicales libres, que son ocupados por grupos sulfhídricos que hacen tornar un poco amargo el producto (Park y Drake, 2016).

\section{Modelado y simulación de la evaporación como operación unitaria}

El proceso de concentración por evaporación puede ser analizado haciendo uso de la modelación matemática, con el fin de facilitar su comprensión y representación (Núñez et ál., 2012). Existen una gran variedad de modelos matemáticos que representan el comportamiento de un tren de evaporación (véase Cadet et ál., 1999; Riverol y Napolitano, 2000; Runyon et ál., 1991; Tonelli et ál., 1990). Normalmente, la principal diferencia entre los distintos modelos matemáticos es el conocimiento heurístico utilizado para su desarrollo. Las hipótesis incluidas en estos modelos están generalmente relacionadas con el cálculo de propiedades termofísicas o algún parámetro como el coeficiente global de transferencia de calor, área de transferencia, calor latente de evaporación o masa en cada efecto (Miranda y Simpson, 2005; Yadav y Jana, 2010).

En la actualidad, el tema de la simulación de procesos y su optimización van de la mano, lo que repercute directamente sobre los costos. El uso de vapor vivo hace alusión al vapor que debe suministrarse a la solución que va a ser concentrada. Este vapor se produce en una caldera de vapor y determina la economía del proceso, ya que el costo de producción de este vapor vivo representa una parte fundamental de los costos operativos del sistema (y lo que se busca es que dicha cantidad disminuya).

El vapor que la solución genera al evaporar el solvente volátil presente se puede aprovechar usando dos métodos: la recompresión de vapor térmico (en el que el vapor vivo a alta presión se utiliza para impulsar el eyector de chorro de vapor y aumentar la presión y la temperatura del vapor producido en el evaporador) y recompresión mecánica del vapor (en el que el aumento de la T y P se logran usando un compresor o un ventilador) (Gourdon y Mura, 2017). Otra opción para disminuir costos es el diseño del evaporador teniendo en cuenta los tubos al interior del tren de evaporación, así como su diámetro y el costo de la energía (Díaz, Galván, Ramos y Castillo, 2013).

Los trabajos pioneros basaron su optimización en una integración energética para aprovechar al máximo la energía liberada por el sistema y determinar de manera rigurosa el área de transferencia de calor.

El cálculo del área de transferencia de calor de manera rigurosa es tratado por Alí, Elhassan, Mustafa y Abdelrhman (2016), con el fin de obtener el largo y el diámetro de los tubos que serán instalados en el evaporador. Este estudio realizó una integración energética en una fábrica de papel por un método llamado heatsep, el cual consiste en el aislamiento de todos los proceso que requieran transferencia de calor en una caja negra, la cual es resuelta en dos fases: diseño de la configuración básica de la planta (específicamente, dispositivos de transferencia de calor) y creación de una red de 
intercambio de calor entre las corrientes, con el fin de aprovechar las cargas térmicas de cada fluido y realizar la integración energética. Al ejecutar la optimización del tren de evaporación se usó únicamente vapor vivo en el primer efecto del tren, y usando el vapor producido al concentrar la solución se obtuvo el medio de calentamiento para el siguiente efecto; para esto se utilizó un ambiente de simulación gráfico (que hace parte de MATLAB) de sistemas genéricos que realizan un proceso de integración de calor, con lo que se determina la mejor configuración que puede tener el tren de evaporación.

La programación no lineal es utilizada para realizar la optimización del arranque de una estación de evaporación. En la planta de evaporación se controlan las siguientes variables: los niveles de cada evaporador (que deben permanecer en $62 \%$ ), la presión en cada uno de los evaporadores y la concentración de salida del producto en el último evaporador al 82 \%. En el proceso se siguen tres criterios: garantizar que el sistema alcance un equilibrio en el menor tiempo posible, usar la menor cantidad de vapor fresco y seguir las especificaciones del sistema (nivel del evaporador, presión y concentración final de producto). Aplicando un proceso de optimización dinámica, en el que solo se deja como grado de libertad la cantidad de vapor que ingresa al sistema, e instalando tres controladores (de nivel, de presión y de concentración) se realiza el primer modelamiento de la optimización. Posteriormente se tiene en cuenta el tiempo de arranque, la cantidad de vapor requerido para dicho proceso y la representación matemática que se realizó en función de los costos, con la que se establecen las condiciones óptimas para realizar el arranque de dicha planta (de Prada, Cristea y Rosano, 2007).

El trabajo de Pitarch, Palacín, De Prada, Voglauer y Seyfriedsberger (2017b) hace especial énfasis en la obtención de parámetros reconciliados como punto de partida para realizar una optimización del proceso. La reconciliación de datos (DR) es definida usualmente como un sistema para resolver inconsistencias entre las mediciones de la planta y los balances de masas (entre otros balances, como balances de energía o de componentes) en toda la planta.

Otra de las aplicaciones de las simulaciones es la determinación preliminar de la forma cómo se comportará el proceso en una fábrica; de esta manera se predicen costos y cantidades que pueden ser tratadas en la planta sin poner en riesgo la integridad de la misma, y se presentan diferentes alternativas para realizar los cálculos de manera sistemática (omitiendo los largos y tediosos cálculos hechos a mano).

En un método corto para el diseño de un sistema de evaporación de múltiple efecto se hace énfasis en que el método no es iterativo y que se obtiene una respuesta inmediata. Los datos iniciales que se requieren en este caso son: número de efectos, tipo de sistema de alimentación (paralelo, contracorriente, etc.), flujo de la alimentación, presión y/o temperatura del vapor vivo, presión o temperatura de saturación del último efecto, valores de los coeficientes globales de transferencia de calor para cada uno de los efectos, concentración de la alimentación y producto final. Todos estos datos se necesitan para obtener la cantidad de vapor vivo requerido (Carrizales, 2010).

Belghaieb, Aboussaoud, Abdo y Hajji (2010) simularon un evaporador de triple efecto perteneciente a una industria de ácido fosfórico, la cual usa agua destilada 
proveniente de un proceso de desalinización de agua de mar. Llama la atención que para solucionar el problema de formación de incrustaciones en el evaporador de película descendente se hizo uso de un inhibidor, el cual retarda la precipitación de la sal. La simulación (realizada en el software Aspen Plus) encontró la cantidad de calor necesaria para realizar la evaporación y la cantidad de vapor producido, la tasa de evaporación por efecto y la cantidad de agua destilada producida. Se simularon evaporadores (tanques flash) y condensadores (calentadores).

Los evaporadores de múltiple efecto han sido estudiados por su utilidad en la industria. Usando de una hoja electrónica Rangel y Argoti (1999) calcularon la entalpia de la solución en función de la concentración y la temperatura del fluido. Asimismo, gracias al uso de esta herramienta se puede calcular el aumento del punto de ebullición de la solución producto del aumento de la concentración del soluto, haciendo que la temperatura de ebullición sea superior a la del agua pura; con estos datos se procede a realizar (por medio de criterios de inicialización, como el cambio de temperatura entre cada efecto y las condiciones de salida del producto concentrado) el cálculo de los requerimientos de vapor vivo y el número de efectos, lo cual permite conocer el costo de la operación.

La concentración de hidróxido de sodio que debe efectuarse, luego de realizar el proceso cloro-alcalí, es la electrólisis del agua salada para producir cloro y soda cáustica, además de hidrógeno. La solución emergente de este proceso es $\mathrm{NaOH}$ con diferentes concentraciones (entre 11 y $25 \% \mathrm{~W}$ ), que debe ser concentrada hasta llegar a $50 \%$ utilizando un evaporador de triple efecto (Vazquez-Rojas, Garfias-Vásquez y Bazua-Rueda, 2018). Se utilizó el software Aspen Plus y la base de datos SYSOP15M. En la Figura 7 se presenta la simulación realizada, la que contiene parámetros del modelo ENTRL-RK ajustados a partir de datos publicados por Silcock (1977), modelo que es comúnmente usado para soluciones electrolíticas (estos datos también fueron usados por Chen y Evans [1986], Chen y Song [2004] y Song y Chen [2009]).

\section{Figura 7}

Diagrama de flujo de la modelación en Aspen Plus de un tren de evaporatores de triple efecto.

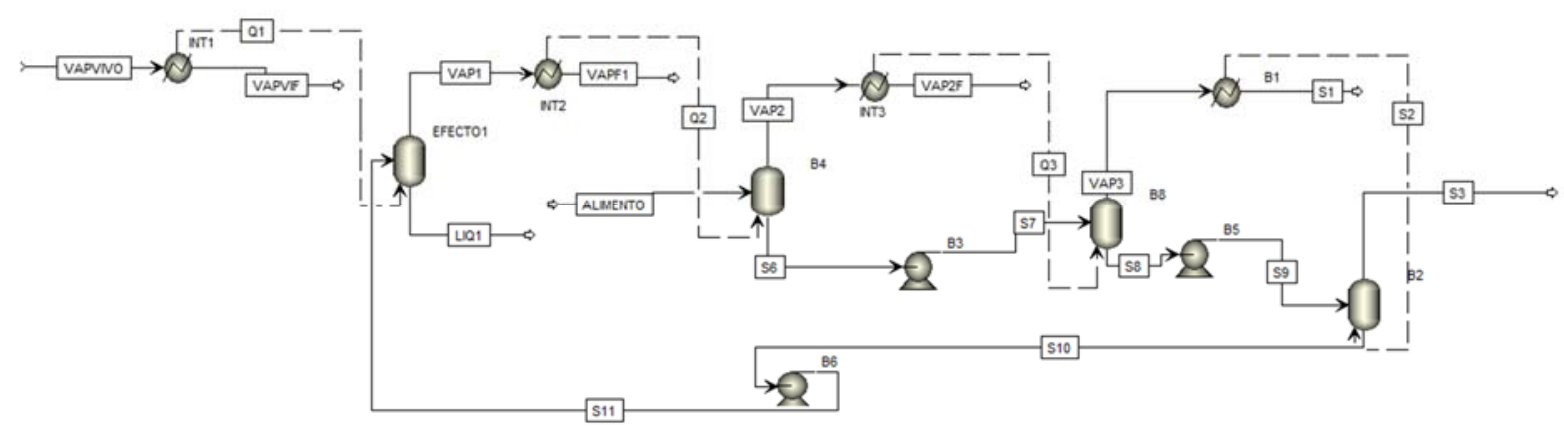


Igualmente, la simulación permite analizar la viabilidad económica de proceso. Alí et ál. (2016) trataron este tema en el caso de la producción de agua potable por medio del uso de la desalación del agua de mar. La herramienta que se empleó en este caso fue Aspen Hysys, en la que usando solo tres parámetros (temperatura del vapor, temperatura de salida en el último efecto y coeficiente global de transferencia de calor del primer efecto) se puede conocer los costos que acarrea la operación. El estudio concluyó que el costo de producción estimado para un metro cúbico de agua potable era de 5 dólares aproximadamente y la inversión realizada tendría un periodo de recuperación de 7 años (Alí et ál., 2016), lo cual hace que esta tecnología no sea rentable.

\section{Nuevas tecnologías empleadas en la evaporación}

El principal desafío que tiene la industria de la evaporación es hacer el proceso más económico, además de buscar nuevas tecnologías con las que se pueda trabajar a temperaturas ambiente, con el fin de no alterar las propiedades fisicoquímicas del alimento a concentrar, como es el caso de materiales termosensibles.

Un método alterno a la evaporación convencional es el uso de salmuera, la cual por principio osmótico extrae el contenido de agua presente; posteriormente, por medio de una membrana se separan la solución de extracción del líquido concentrado. El proceso se realiza a presiones atmosféricas y temperatura ambiente, evitando que las soluciones que son susceptibles a los cambios de temperatura, como es el caso de las frutas, no sufran reacciones de pardeamiento ni se degraden sus propiedades (color, olor, sabor o aromas). Forero y Vélez (2013) realizaron un diseño experimental de superficie de respuesta, en la que se varió la cantidad de salmuera, la cantidad de jugo y la temperatura (en la cual sucedió la evaporación) y se obtuvo valores de concentración de la solución problema en ${ }^{\circ}$ BRIX y la cantidad de flujo que resultó concentrada. Posteriormente se aplicó una prueba F para identificar si los parámetros que se están variando influyen de manera significativa en las variables de salida.

Otro de los métodos no convencionales es el uso de ondas ultrasónicas para realizar la excitación de las moléculas, lo que genera la evaporación a temperaturas relativamente bajas, como lo muestra la investigación de Dehbani y Rahimi (2018). El método consiste en hacer pasar la solución por una superficie rugosa en la que se encuentran interconectados transductores que generan ondas ultrasónicas, las cuales trasmiten energía a la película que desciende por una superficie previamente instalada. Gracias a la energía suministrada en la película se comienza a formar el fenómeno de la cavitación.

La exposición del fluido al ultrasonido crea una turbulencia a microescala. Por lo tanto, gradualmente aumenta la turbulencia a microescala a través de la mayor parte del fluido. Luego, a medida que pasa el tiempo, aumenta la turbulencia a microescala a través del fluido que se desborda en la placa inclinada equipada con transductores 
ultrasónicos y ayuda a mejorar la eficiencia. Por lo tanto, la tasa de evaporación mejora. (Dehbani y Rahimi, 2018, p. 690)

La temperatura juega un papel fundamental a la hora de realizar este proceso, ya que a más $50{ }^{\circ} \mathrm{C}$ el efecto de aplicar el ultrasonido es negativo y no contribuye a la evaporación debido a la formación de burbujas más grandes y numerosas, las que se acumulan en la sección de prueba, amortiguando el efecto de las vibraciones ultrasónicas y reduciendo el efecto de mezcla de la misma. Gracias a este experimento se determinó que $40^{\circ} \mathrm{C}$ es la temperatura óptima en la que se debe realizar este proceso, pues es la temperatura en la que la turbulencia aumenta. Como se explicó, uno de los mayores problemas de la evaporación es la obtención de la energía requerida para evaporar la solución. Al respecto, Liu, Xu y Wang (2017) proponen el uso de la energía solar como fuente energética para realizar dicha operación unitaria, haciendo uso de paneles solares compuestos de láminas fototérmicas para capturar la energía lumínica. Se estudiaron tres tipos de agentes fototérmicos: material a base de carbono, metales plasmónicos y materiales semiconductores, los cuales son estudiados en detalle en el artículo.

El problema del uso de esta energía radica en la gran área superficial que el sistema debe tener para capturar la energía. Es por esto que Papikyan, Gevorgyan, Mangasaryan y Gladyshev (2018) desarrollaron un método en el que se hace uso de paneles solares con una película de Cd-Te, con lo que se capturó un 35 \% más de energía lumínica. El proceso consiste en utilizar un sistema de evaporación flash con una solución en la que se encuentran los componentes de la película. El grosor de las películas de Cd-Te y su tiempo de deposición fueron 1-2 $\mu \mathrm{m}$ y 30-50 min, respectivamente.

El sistema de humificación deshumidificación es un procedimiento que permite la reducción del consumo energético producido en la evaporación. Este es un método promisorio que está siendo aplicado en procesos industriales como la desalinización y tratamiento de aguas residuales, así como en materiales que son termosensibles, gracias a la capacidad que tiene el aire de transportar gran cantidad de vapor de agua a temperaturas ambiente. El principio de funcionamiento radica en la capacidad que tiene el aire seco de atrapar humedad de una corriente de agua caliente; parte de esta agua se evapora y se transfiere y humecta al aire seco, lo que transporta vapor de agua en una corriente de aire a temperaturas ambiente. Gracias al uso de esta tecnología no solo se reduce el costo de operación, sino también los costos fijos, ya que solo se tiene una cámara de evaporación. Xin, Yang, Tingfen, Xiang y Weihong (2017) y Wang, Li y Lakerveld (2018) hacen un barrido sobre los desarrollos recientes de procesos de concentración, particularmente en la industria de cristalización de fármacos; el uso de microondas como fuente de energía para la evaporación del solvente de la solución se presenta como una alternativa que logra una rápida evaporación, lo que conduce a una sobresaturación instantánea y, por ende, a una producción de cristales de tamaño reducido. Se enuncia el uso de nanopartículas de plata que actúan con sitios de nucleación para cristales de aminoácidos, como acetaminofén, glicina y arginina.

\section{CONCLUSIONES}


La evaporación es una operación industrial con múltiples variables operativas y estructurales; dentro de las variables estructurales se encuentra el tipo de evaporador y algunos aspectos del diseño conceptual de la operación (de película descendente, mamita abierta, múltiple efecto, calandria vertical, entre otros). Estos equipos son empleados en diferentes industrias para, por ejemplo, desalinización de aguas, concentración de soluciones compuestas por $\mathrm{NaOH}$, concentración de productos en la industria farmacéutica o alimentos (producción de azúcar o elaboración de jaleas de todo tipo de frutas). El tipo de evaporador es escogido de manera minuciosa de acuerdo a las necesidades $\mathrm{y}$, sobre todo, a la sensibilidad de los materiales que serán concentrados, así como para garantizar la mayor eficiencia del proceso, lo que se traduce en ahorros en el consumo de energía de la operación.

La evaporación tiene un papel fundamental en la industria, ya que esta tecnología reduce considerablemente el costo de transporte de los productos, aunque sin dejar de lado la preservación de las propiedades organolépticas del mismo.

Las investigaciones sobre evaporación están enfocadas en la disminución del costo de dicha operación unitaria para hacerla competitiva en el mercado. Se han logrado importantes mejoras, como aumentar la turbulencia del fluido, precalententar la solución, usar trenes de evaporación, integración energética, disminuir las incrustaciones en los evaporadores, entre otras. Por otra parte, con la ayuda de sistemas complejos de optimización se han obtenido las configuraciones más adecuadas para cada sistema en particular.

Igualmente, la simulación es una herramienta que está siendo usada en todas las industrias, incluyendo la representación de procesos de evaporación, en las que se pueden probar diferentes configuraciones de los equipos sin tener la necesidad de poner en riesgo la integridad de una planta.

El artículo muestra que la industria viene mejorando sus procesos, desde agregar serpentines, modificar el espaciado entre los tubos en la sección de intercambio de calor o agregar platos de alimentación para mejorar la humectación, hasta buscar métodos alternativos en los que se introducen tecnologías como el ultrasonido, humidificación y deshumidificación. Entre los métodos alternativos se encuentra el aprovechamiento de energías limpias para realizar la evaporación del solvente, como mejorar las técnicas de captura en energía lumínica o integrar energías alternativas.

Adicionalmente, se encontró que se debe desarrollar mecanismos que permitan trabajar con materiales termosensibles, así como realizar simulaciones mucho más robustas que permitan un acercamiento más real a la industria.

\section{SÍMBOLOS Y ABREVIATURAS}

2D: dos dimensiones

3D: tres dimensiones 
Ayala-Padilla, Pabón-Beltrán y Correa-Mahecha

Re: número de Reynolds

Q: calor

DR: reconciliación de datos

$\% \mathrm{~W}$ : porcentaje en peso

${ }^{\circ} \mathrm{Bx}$ : grados brix

$\mathrm{T}$ : temperatura

P: presión

\section{Referencias}

Alí, E., Elhassan, A., Mustafa, H., y Abdelrhman, O. (2016). Design configuration and simulation of A multi effects evaporator for A desalination plant. International Journal of Scientific \& Technology Research, 5(9), 118-120.

Avalo, B., Pérez, S., y Tovar, M. (2009). Caracterización preliminar del proceso de concentración del jugo natural de naranja en un evaporador de tres efectos. Inteciencia, 34(11), 784-790.

Ayala (2018). Planteamiento metodológico para el diagnóstico de las condiciones de operación de planta de evaporación bajo condiciones de diseño establecidas por el fabricante, 128-134.

Belghaieb, J., Aboussaoud, W., Abdo, M., y Hajji, N. (2010). Simulation and optimization of a triple-effect distillation unit. https://pdfs.semanticscholar.org/4a0a/9611c8776a67848b36d9c8db02c264a 45006.pdf? ga=2.90174875.1676902329.1549299583$\underline{1798874874.1549299583}$

Brennan, J., Butters, J., y Lilly, A. (1998). Las operaciones de la ingeniería de los alimentos (3a ed.). Acribia S.A.

Cadet, C., Toure, Y., Gilles, G., y Chabriat, J. (1999). Knowledge modeling and nonlinear predictive control of evaporators in cane sugar production plants. Journal of Food Engineering, 40(1/2), 59-70.

Carrizales, R. (2010). Cálculo de evaporadores de múltiple efecto, un método simplificado. Tlatemoani-Revista Académica de Investigación, 2, 1-11.

Chantasiriwan, S. (2018). Online determination of heat transfer coefficients in sugar juice evaporation process. Journal of Food Engineering, 230, 63-71. DOI: 10.1016/j.jfoodeng.2018.02.021

Chen, C., y Evans, L. (1986). A local composition model for the excess gibbs energy of aqueous electrolyte systems. AIChE Journal, 32(3), 444-454. DOI: 10.1002/aic.690320311

Chen, C., y Song, Y. (2004). Generalized electrolyte-NRTL model for mixed-solvent electrolyte systems. AIChE Journal, 50(8), 1928-1941. doi:10.1002/aic.10151 
Chen, J., Zhang, J., Hu, Z., y Ma, Z. (2017). Falling film transitions on horizontal enhanced tubes: Effect of tube spacing. Procedia Engineering, 205, 15421549. DOI: $10.1016 /$ j.proeng.2017.10.242

De Prada, C., Cristea, S., y Rosano, J. (2007). Optimal start-up of an evaporation station. IFAC Proceedings 40(5), 113-118. DOI: 10.3182/20070606-3-MX-2915. 00137

Dehbani, M., y Rahimi, M. (2018). Introducing ultrasonic falling film evaporator for moderate temperature evaporation enhancement. Ultrasonics Sonochemistry, 42, 689-696. DOI: 2126/10.1016/j.ultsonch.2017.12.016

Demesa, N., Huicochea, A., Hernandez, J., y Siqueiros, J. (2017). Heat transfer coefficients for evaporator with nested helical coils. International Journal of Thermal Sciences, 121, 390-397. DOI: 2126/10.1016/j.ijthermalsci.2017. 08.002

Díaz, C., Galván, E., Ramos, E., y Castillo, F. (2013). Metodología para el diseño óptimo de evaporadores de película descendente. Avances en Ciencias $e$ Ingeniería, 4(3), 50-52.

Forero, F., y Vélez, C. (2013). Optimización de la concentración por evaporación osmótica del jugo de maracuyá (Passifora edullis). Dyna, 80(179), 90-98.

García, H., Peña, A., López, R., Duran, E., y Olvera, G. (2009). Desarrollo de un sistema de evaporación y concentración de jugos de múltiple efecto para mejorar la eficiencia térmica y la productividad y disminuir el impacto ambiental en la producción de panela.

http://bibliotecadigital.agronet.gov.co/bitstream/11348/4735/1/Microsoft $\% 2$ 0Word-MINAGRICULTURA.-_proyecto\%20Pa.pdf

Gautami, G., y Khanam, S. (2012). Selection of optimum configuration for multiple effect evaporator system. Desalination, 288, 16-23. DOI: 10.1016/j.desal. 2011.12.005

Geankoplis, C. (1998). Procesos de transporte y operaciones unitarias (3a ed.). Continental S. A.

Gourdon, M., y Mura, E. (2017). Performance evaluation of falling film evaporators in the dairy industry. Food and Bioproducts Processing, 101, 22-31. DOI: 10. 1016/j.fbp.2016.10.004

Guerrero, C., Escobar, S., y Ramírez, D. (2005). Manejo de la salinidad en aguas asociadas de producción de la industria petrolera. Ingeniería e Investigación, 25(3), 27-33.

Harrison, R. (2014). Project management procedures needed to design the newest four phosphoric acid evaporators in North America. Procedia Engineering, 83, 462-468. DOI: 2126/10.1016/j.proeng.2014.09.079

Heluane, H., Blanco, A., Hernandez, M., y Bandoni, J. (2013). Planteamiento operativo de redes de evaporación. Iberoamerican Journal of Industrial Engineering, 5(9), 358-375.

Jastoria (2015) Thin film evaporator [imagen original en archivo PNG]. https://commons.wikimedia.org/wiki/File:Thin_Film_Evaporator.png

Jyoti, G., y Khanam, S. (2014). Simulation of heat integrated multiple effect evaporator system. International Journal of Thermal Sciences, 76, 110-117. DOI: 10.1016/j.ijthermalsci.2013.08.016 
Liu, G., Xu, J., y Wang, K. (2017). Solar water evaporation by black photothermal sheets. Nano Energy, 41, 269-284. DOI: 10.1016/j.nanoen.2017.09.005

Mendieta, O., Rodríguez, G., y García, H. (2008). Comparación de las alternativas de producción de panela en la hoya del río Suárez. Colombia: Corpoica.

Miranda, V., y Simpson, R. (2005). Modeling and simulation of an industrial multiple effect evaporator: tomato concentrate. Journal of Food Engineering, 66(2), 203-210.

Morison, K. (2015). Reduction of fouling in falling-film evaporators by design. Food and Bioproducts Processing, 93, 211-216. DOI:10.1016/j.fbp.2014.10.009

Mura, E., y Gourdon, M. (2017). Pressure drop in dairy evaporators: experimental study and friction factor modelling. Journal of Food Engineering, 195, 128-136. DOI: 10.1016/j.jfoodeng.2016.09.020

Núñez, Á, Tarifa, E., y Farfán, N. (2012). Simulación estacionaria de un evaporador de doble efecto para jugo de tomate. Avances en Ciencias e Ingeniería, 3(4), 19 .

Padilla, B. (2013). Diseño de un evaporador tipo marmita con agitador y serpentín interno para la elaboración de diversos alimentos en planta piloto (tesis de pregrado). Escuela de Ingeniería Química, Facultad de Ingeniería, Universidad san Carlos de Guatemala, Ciudad de Guatemala, Guatemala.

Papikyan, A., Gevorgyan, V., Mangasaryan, y Gladyshev, P. (2018). Characterization of vacuum flash evaporated CdTe thin films for solar cell application. Journal of Physics: Conference Series, 945(1), 1-4. DOI: 10.1088/1742-6596/945/ $1 / 012013$

Park, C., y Drake, M. (2016). Condensed milk storage and evaporation affect the flavor of nonfat dry milk. Journal of Dairy Science, 99(12), 9586-9597. DOI: 10.3168/jds.2016-11530

Pitarch, J., Palacín, C., de Prada, C., Voglauer, B., y Seyfriedsberger, G. (2017). Optimisation of the resource efficiency in an industrial evaporation system. Journal of Process Control, 56, 1-12. DOI: 2126/10.1016/j.jprocont.2017.04. 002

Process Solution and Equipment (2018). Manual de operación \& NBSP; tren de evaporación triple efecto (control manual) REF. TE-M. Bogotá D. C.: PS\&E.

Process Solutions and Equipment (2018). Guía de laboratorio tren de evaporadores (control manual) REF. TE-M. Bogotá D. C.19-20

Rangel, H., y Argoti, A. (1999). Cálculo numérico de un evaporador químico sobre una hoja electrónica. Revista de Ingeniería e Investigación Universidad Nacional de Colombia, 44, 48-55.

Riverol, C., y Napolitano, V. (2000). Non-linear Control of an evaporator using an error trajectory technique. Journal of Chemical Technology and Biotechnology, 75, 1047-1053.

Rojero, M., García, J., Serrano, B., Félix, M., y Ríos, G. (2008). Simulación de un evaporador de doble efecto. caso de estudio: jarabe de azúcar. Revista Investigación Científica, 4(2), 1-6.

Runyon, C., Rumsey, T., y McCarthy, K.L. (1991). Dynamic simulation of a non-linear model of double effect evaporator. Journal of Food Engineering, 14, 185-201. 
Sánchez, J., y Pérez, O. (1976). Construcción de plantas desaladoras tipo evaporación instantánea a base de etapas estructural mente independientes. Desalination, 19(1-3), 269-282.

Scopus (2018). Evaporation Train search.

https://ezproxy.uamerica.edu.co:2080/term/analyzer.uri?sid=bf23ea1e9b810 46d89876689cf8b2aed\&origin=resultslist\&src=s\&s=TITLE-

ABSKEY\%28evaporator+train\%29\&sort=plff\&sdt=sisr\&sot=b\&sl=31\&cou

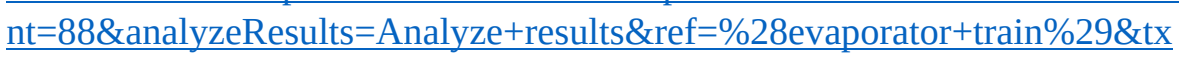
Gid=fd2ab57d5ce206064a61f68494e5f970

Seader, J., Henley, E., y Roper, D. (2011). Separation process principles chemical and biochemical operations (3a ed.). John Wiley \& Sons, Inc.

Semiat, R. (2001). Desalination education capacity in Israel. Desalination, 141(2), 191198. DOI: 10.1016/S0011-9164(01)00403-9

Silcock, H. (1977). Generalized wreath products and the lattice of normal subgroups of a group. Algebra Universalis, 7(1), 361-372. DOI: 10.1007/BF02485445

Song, Y., y Chen, C. (2009). Symmetric electrolyte nonrandom two-liquid activity coefficient model. Industrial \& Engineering Chemistry Research, 48(16), 7788-7797. DOI: 10.1021/ie9004578

Srivastava, D., Mohanty, B., y Bhargava, R. (2016). Characterization of evaporator scale of indian sugar industry. Applied Thermal Engineering, 98, 894-899. DOI: 10.1016/j.applthermaleng.2015.12.130

Tonelli, S., Romagnoli, J., y Porras, J. (1990). Computer package for transient analysis of industrial multipleeffect evaporators. Journal of Food Engineering, 12(4), 267-281.

Vazquez-Rojas, R., Garfias-Vásquez, F., y Bazua-Rueda, E. (2018). Simulation of a triple effect evaporator of a solution of caustic soda, sodium chloride, and sodium sulfate using aspen plus. Computers and Chemical Engineering, 112, 265-273. DOI: 10.1016/j.compchemeng.2018.02.005

Wang, J., Li, F., y Lakerveld, R. (2018). Process intensification for pharmaceutical crystallization. Chemical Engineering and Procesasen-Process Intensification, 127, 111-126. DOI: 10.1016/j.cep.2018.03.018

Xin, H., Yang, L., Tingfen, K., Xiang, L., y Weihong, L. (2017). Thermal investigation and performance analysis of a novel evaporation system based on a humidification-dehumidification process. Energy Conversion and Management, 147, 108-119. DOI: 10.1016/j.enconman.2017.05.036

Yadav, P., y Jana, A. (2010). Simulation and Control of a Commercial Double Effect Evaporator: Tomato Juice. Chemical Product and Process Modeling, 5(1), http://www.bepress.com/cppm/vol5/iss1/6. DOI: 10.2202/1934-2659.1443. 